\title{
COMMISSION 47: COSMOLOGY (COSMOLOGIE)
}

\author{
PRESIDENT: Alexander Szalay \\ VICE-PRESIDENT: John Peacock \\ ORGANIZING COMMITTEE: Y. Chu L. da Costa, J. Einasto, G. Ellis, \\ D. Koo, S. Lilly, J. Narlikar, P. Shaver, R. Webster, S. White \& A. Wolfe
}

\section{HIGH PRECISION COSMOLOGY: THE AGE OF MEGA-SURVEYS}

Cosmology is one of the most dynamically evolving areas of astrophysics today. Twenty years ago the estimates of the amplitude of the primordial fluctuations were about $10^{-3}$, almost a factor of 100 off of today's measurements. Ten years ago we could only hope for high precision measurements of large scale structure, there were less than 5000 redshifts measured, and only a handful of normal galaxies with $z>1$ were known. Computer models of structure formation had just begun to consider non-power-law spectra based on physical models like hot/cold dark matter. As a consequence there was considerable freedom in adjusting parameters in the various galaxy formation scenarios. In contrast, many of today's debates are about factors of 2 and soon we will be arguing about $10 \%$ differences. The Harrison-Zeldovich shape of the primordial fluctuation spectrum, first derived from philosophical arguments can now be quantified from detections of fluctuations by COBE. The number of available redshifts is beyond 50,000 , and soon we will have redshift surveys surpassing 1 million galaxies. $\mathrm{N}$-body simulations are becoming more sophisticated, of higher resolution, and incorporating complex gas dynamics.

There are many large digital survey projects under way, which together will create a unified multi-wavelength view of the Universe. Efforts include 2MASS, SDSS, DPOSS, FIRST, NVSS, COBE, MAP, GSC-II, ROSAT, GALEX, and GLAST, with wavelengths from gamma-rays and X-rays through UV, optical, near-IR to microwave and radio. The projects listed here will provide (almost) all-sky information in more than 15 different bands. These have all been designed to be homogeneous, well calibrated and have a good control over systematics. The catalogs will typically contain over 10-100 million objects each, and will be multiple Terabytes in size. These new surveys will revolutionize the field of cosmology, increasing the amount of information available to researchers by several orders of magnitude. The first few of these are already either taking data or will soon be doing so. These include the Sloan Digital Sky Survey and the 2dF redshift survey. They will both produce detailed 3D maps of the galaxy distribution, with sample sizes reaching close to the million. The analysis of these mega-surveys will require new, different approaches: the accuracy of the results will not be limited by the small sample size, for the first time in cosmology, but rather will be driven by systematics.

The unprecedented number of new observations under way promises that over the next decade we will gain a clear understanding of the shape and evolution of the primordial fluctuation spectrum, understand from first principles how galaxies were formed, and make quantitative comparisons and tests to differentiate among the various galaxy formation scenarios. So far the CMB experiments have measured fluctuations mostly on scales above 300 $\mathrm{Mpc}$, where the shape is expected to be primordial. Galaxy surveys were mostly sensitive to the regime below $100 \mathrm{Mpc}$, where strong clustering evolution does leave a significant imprint on the spectrum. The least known, and at the same time the most interesting part of 
the fluctuation spectrum is on scales between $100-300 \mathrm{Mpc}$, where primordial sound waves may have left a detectable imprint, like the Doppler peaks in the CMB fluctuations. This regime will soon be well studied by both CMB experiments and redshift surveys. Other surveys are probing the Universe to unprecedented depths and gravitational lensing is well on its way to be used as a practical tool to measure the amount of dark matter directly. In the following sections we summarize the recent developments in these rapidly evolving subfields, all tied together by our drive for measuring the fundamental cosmological quantities to unheard levels of precision.

\section{MICROWAVE BACKGROUND FLUCTUATIONS}

We are in the middle of a remarkable growth period for CMB anisotropy research, in theory, experiment and in data analysis techniques. The major arena for confronting theory and observation continues to be the angular power spectrum of temperature fluctuations, $\left.C_{\ell} \sim<\left|T_{e l l m}\right|^{2}\right\rangle$, a function of the multipole number in a spherical harmonic expansion $\ell$ (angular wavenumber at high $\ell$ ). Beginning with the COBE anisotropy at $\ell \lesssim 20$, solidified now with 4 years of data fully analyzed, we have seen a steady stream of solid detections up to $\ell \sim 600$, and important upper limits beyond that. The data that currently exists and has been analyzed, leads to highly significant constraints on the character of the cosmic structure formation models and the cosmological parameters that define it. Within the inflation paradigm, one of the parameters best determined is the slope of the primordial power spectrum (nearly scale invariant within about $10 \%$, as is predicted in the simplest inflation models). With CMB data alone, extreme open models are ruled out. Combining CMB and large scale structure data favours models with a sizeable cosmological constant or other form of unclustered energy, and even strongly rules out open models relative to these. The results are highly compatible with the supernova data (e.g., Bond \& Jaffe 1999).

Many quantitative (but idealized) forecasts were made of how well CMB experiments using ground-based interferometry and long duration balloons over the next few years, the MAP satellite, to be launched by NASA in 2000 , and the Planck satellite, to be launched by ESA in 2007, could ultimately do in improving $C_{\ell}$ determinations, with the byproduct percent level accuracy in a half dozen or so of the cosmological parameters defining the dark-matter dominated inflation-based standard model of structure formation. These exercises also explored the limitations that Galactic and extragalactic foregrounds would likely impose on accuracy (apparently not overly restrictive), enhancing the case for their successful funding, and showed that near degeneracies would exist among certain parameters, which non CMB-experiments would be required to break (e.g., the total cosmological density parameter and the cosmological constant). The nature of the polarization of the CMB from tensor perturbations induced by gravity waves as well as by the familiar scalar perturbations that give rise to structure was further explored theoretically and a number of experiments to detect it are now planned, fueled by the realization that polarization can help to break the degeneracies, especially to constrain the degree to which radical departures from scale invariance are possible.

Since the typical fractional temperature anisotropy $C_{\ell}^{1 / 2}$ is in $10^{-5}$ units, each new detection has been hard won; those in the past few years have been reported by groups under the acronyms BAM $(\ell \sim 30-90)$, Python $(\ell \sim 60-240)$, QMAP $(\ell \sim 40-180)$, TOCO (or MAT) $(\ell \sim 100-400)$, CAT $(\ell \sim 340-720)$ and OVRO $(\ell \sim 360-750)$, an upper limit by SuZIE $(\ell \sim 1400-3700)$ and an apparent detection by Viper $(\ell \sim 300)$. Combined with the plethora of results from pre-1997 experiments, including recent in-depth studies of Saskatoon $1995(\ell \sim 60-400)$ and MSAM $(\ell \sim 80-340)$, the classic $C_{\ell}$ plot is overwhelmed by the observations. However, an optimal power spectrum determination, assuming the underlying anisotropies are Gaussian-distributed, shows a clear peak, roughly at $\ell \sim 200$, with a drop beyond this (Bond, Jaffe \& Knox 1999). This shape is in good accord with the (highly refined) $C_{\ell}$ predictions of the standard inflation-based model: large deviations are ruled out, leading to the parameter estimates described above. Even the COBE data by itself has continued to be fruitful, prompting a renewed look for example on 
how large the Universe must be, using CMB constraints on the size of models with compact topology with negative curvature.

\section{PROBING THE DISTANT UNIVERSE: THE HUBBLE DEEP FIELD}

The Hubble Deep Field Surveys (North and South, HDF-N and HDF-S; Williams et al. 1996,2000 ) have become two of the most intensively studied patches of sky, and a focal point for studies of observational cosmology. While hardly the only distant galaxy survey, the sheer volume of high-quality data on the HDFs have made them unique resources. In addition to the original HST programs (150 orbits of HST time each), they have been followed up at nearly every wavelength from both the ground and from space; in each case, the follow-up studies have been among the deepest, most extensive ever carried out. More than 700 spectroscopic redshifts (Cohen et al. 2000) have been measured in and around the HDF-N ( 150 in the central WFPC2 field alone), making it the densest redshift survey ever done. Extremely deep IR imaging from the ground and with NICMOS on board HST (Thompson et al. 1999; Dickinson et al. 2000) have probed the rest-frame optical light from high redshift galaxies, and have enabled the detection (and confirmation) of galaxies at $z>5$. A small number of very faint, "infrared only" objects have been identified, including at least one plausible candidate for an object at $z=12$ (Dickinson et al. 2000). Together, the deep, high angular resolution optical and infrared HST images have been used to follow the morphological evolution of galaxies from $z \approx 3$ to the present, graphically demonstrating the physical growth of galaxies, the maturing of the giant Hubble sequence spirals and ellipticals, and highlighting the predominance of irregular and interacting galaxies at high redshifts.

Used together with other surveys, the HDF has played a key role in tracing the global star formation history of the universe, and also in demonstrating the complications which arise in trying to formulate that very history. The redshift evolution of the volume-averaged, rest-frame UV light from star forming galaxies is one measure of the cosmic star formation history, and the HDF data have played a key role in measuring this at $1<z<5$ (Madau et al. 1996). However, deep mid-infrared and sub-mm surveys of the HDFs (with ISO and SCUBA) have also unveiled a population of high redshift, dust-obscured galaxies which are almost certainly forming stars at prodigious rates, and whose far-IR emission may dominate the global radiative energy density from galaxies. Even with the deepest existing optical and IR data, some of these sub-mm sources have not been identified, providing an ongoing challenge for future investigators.

Thanks to the exceptional supporting data (spectroscopy and imaging), these surveys have allowed astronomers to characterize the $\mu \mathrm{Jy}$ radio sky to an unprecedented degree. Again, a significant fraction $(\sim 20 \%)$ of these radio sources have extremely faint or totally invisible optical and near-IR counterparts (although some do correspond to sub-mm sources), again posing a mystery which remains to be solved.

Perhaps most importantly, the HDF surveys have helped both to spur and to democratize high redshift galaxy studies. The fully reduced HST data were made available to the community shortly after they were taken, and many other investigators have returned the favor, circulating their images, redshifts, and results rapidly for all to use. Among other things, this has fueled a boom in photometric redshift studies (e.g., Connolly et al. 1997; Fernández-Soto et al. 1999) which are essential for understanding the nature of the vast majority of HDF galaxies beyond the spectroscopic limits. The sheer volume of publications using the HDF data or interpreting the evolution seen therein attests to the success of this survey, and its role as a model for future efforts.

\section{GRAVITATIONAL LENSING}

The field of gravitational lensing continues to flourish as one of the principal tools for studying dark matter in a variety of astrophysical settings. In order of descending scale, lensing has been used (i) to limit the cosmological geometry; (ii) to probe the large-scale 
inhomogeneity spectrum; (iii) to study clusters of galaxies; (iv) to probe dark matter in the haloes of individual galaxies; (v) to detect low-mass astrophysical objects, down to planets.

It used to be thought that lensing provided an argument against a large cosmological constant, since the extra high-redshift pathlength in flat lambda-dominated models increases the predicted number of lenses. Since lambda-dominated models have become orthodox through CMB studies and the supernova Hubble diagram, it is interesting to see that these arguments are now inverted: based on better modelling of the lens mass distribution, there is a developing consensus that only lambda-dominated models predict sufficient lensed quasar pairs (Chiba \& Yoshii 1999; Cooray et al. 1999; Quast \& Helbig 1999).

Gravitational lensing is widely expected to lead to large-scale coherent shear of background galaxy images from foreground large-scale structure. Observational detection of this signature is likely to be achieved in $2000 / 2001$, but in the meantime sophisticated theoretical simulations of the expected effect are being produced (Couchman, Barber \& Thomas 1999). A slightly longer-term goal is to detect the effects of large-scale structure lensing on CMB anisotropies. These small effects are interesting in that they will be able to break the cosmological degeneracies expected from CMB data alone (Hu \& Tegmark 1999; Stompor \& Efstathiou 1999). Lensing studies in the strong (multiple-lensing) regime have allowed good understanding of the mass distributions in the central parts of galaxies and clusters of galaxies. Such studies are now starting to realize the power of clusters in particular as natural telescopes, leading to the detection of very low-luminosity high-redshift galaxies (Pelló et al. 1999).

Alexander Szalay

President of the Commission

\section{References}

Bond, J.R. and Jaffe, A.H. 1999, Phil. Trans. R. Soc. Lond. A, 357,57-75.

Bond, J.R. Jaffe, A.H. \& Knox, L. 1999, Ap.J., in press, astro-ph/9808264

Chiba M., Yoshii, Y., 1999, ApJ, 510, 42

Cohen, J.G., Hogg, D.W., Blandford, R., Cowie, L.L., Hu, E.M., Songaila, A., Shopbell, P., \& Richberg, K., 2000, ApJ, submitted

Cooray A.R., Quashnock J.M., Miller M., 1999, ApJ, 511, 562

Connolly, A.J., Szalay, A.S., Dickinson, M., SubbRao, M.U., \& Brunner, R.J., 1997, ApJ, 486,11

Couchman H.M.P., Barber A.J., Thomas P.A., 1999, MNRAS, 308, 180

Dickinson, M., et al., 2000, ApJ, in press

Fernández-Soto, A., Lanzetta, K.M., \& Yahil, A., 1999, ApJ, 513, 34

Hu W., Tegmark M., 1999, ApJ, 514, 65

Madau, P., Ferguson, H.C., Dickinson, M., Giavalisco, M., Steidel, C.C., \& Fruchter, A., 1996, MNRAS, 283, 1388

Pelló R. et al., 1999, A\&A, 346, 359

Quast R., Helbig P., 1999, A\&A, 344, 63

Stompor R., Efstathiou G., 1999, MNRAS, 512, 672

Thompson, R.I., Storrie-Lombardi, L.J., Weymann, R.J., Rieke, M., Schneider, G., Stobie, E., \& Lytle, D. 1999b, AJ, 117, 17

Tucker, G.S., Gush, H.P., Halpern, M., Shinkoda, I., and Towlson, W. 1997, Ap.J.Lett. 475, L73.

Williams, R.E., et al., 1996, AJ, 112, 1335

Williams, R.E., et al., 2000, AJ, in preparation 